\section{Passivrauchen als Ursache von Asthma und Bronchitis}

\section{Die Hälfte aller im Kleinkindalter auftretenden Fälle von Asthma bronchiale, chronischer Bronchitis und „wheezing" könnte durch Passivrauchen verursacht sein.}

\begin{abstract}
$\mathrm{u}$ diesem Ergebnis kommt Peter Gergen von den Centers for Disease Control and Prevention in einer Auswertung der dritten National Health and Nutrition Examinations Survey (NHANES III). In der repräsentativen Stichprobe waren zwischen 1988 und 1994 insgesamt 7680 Amerikaner zu gesundheitlichen Problemen und Ernährungsgewohnheiten interviewt worden.
\end{abstract}

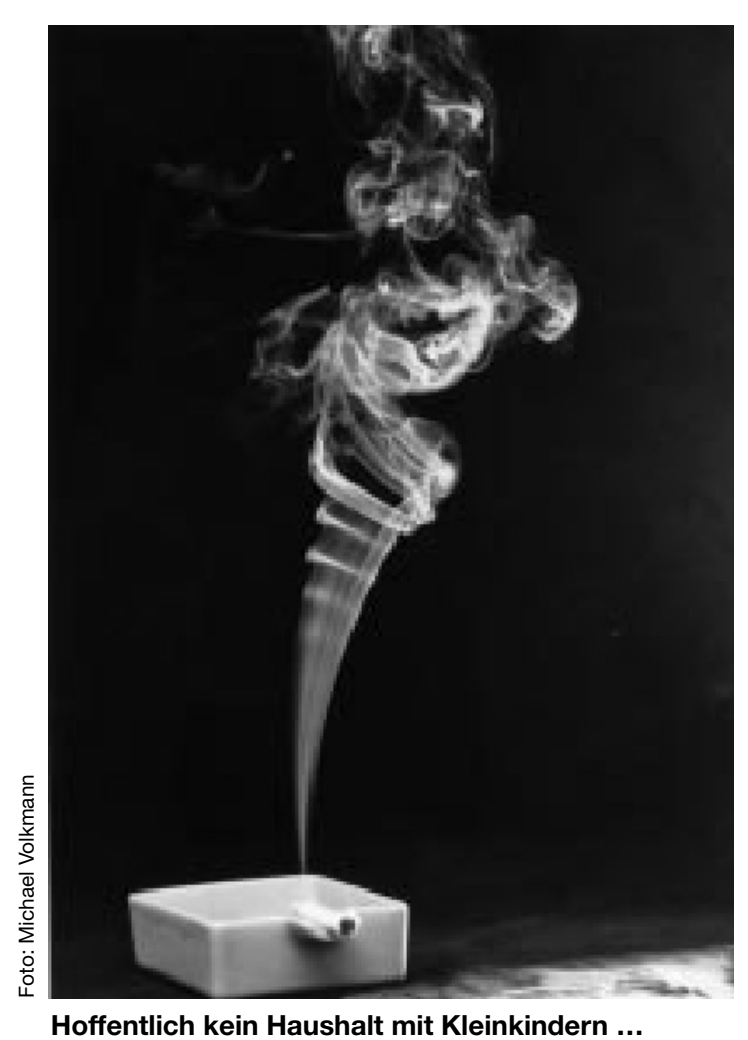

sowie dem entsprechenden Medikamentenverbrauch in Beziehung gesetzt.

Die Belastung von Kleinkindern mit Passivrauchen - die Amerikaner sprechen von Environmental Tobacco Smoke, ETS - war hoch. Insgesamt waren $38 \%$ aller unter 6jährigen dem Zigarettenqualm ihrer Eltern ausgesetzt, bei $23,8 \%$ begann die Belastung bereits intrauterin.

Die Exposition hatte gravierende Folgen für die Gesundheit der Kinder: Chronische Bronchitis, Asthmaerkrankungen oder mehr als drei Keuchanfälle (,Wheezing"-Episoden) traten signifikant $(\mathrm{p}<0,05)$ häufiger in Haushalten auf, in denen täglich mehr als 20 Zigaretten geraucht wurden. Für Asthma scheint bei einem p-Wert von $<0,01$ kein Zweifel am Ergebnis mehr möglich. Hier wurde ein Zusammenhang für die gesamte Altersgruppe bis zum fünften Jahr gefunden. Die Auswirkungen von ETS auf chronische Bronchitis und „wheezing“ konnten dagegen nur für die Altersgruppe von 2 Monate bis 2 Jahre nachgewiesen werden. Hier könnten 40 bis $60 \%$ aller Fälle von Asthma bronchiale, chronischer Bronchitis und ,wheezing“" auf ETS zurückzuführen sein.

Extrapoliert auf die Gesamtbevölkerung der USA

Unter anderem mußten sie angeben, wie viele Zigaretten durchschnittlich im Haushalt geraucht wurden. Mütter wurden gefragt, ob und wieviel Zigaretten sie während der Schwangerschaft geraucht hätten. Die Antworten wurden mit Angaben zu Atemwegserkrankungen der Kinder

\section{Kommentar}

Es ist nicht die erste Untersuchung, die ETS mit Atemwegserkrankungen von Kleinkindern in Verbindung bringt. Dabei zieht sich wie ein roter Faden durch alle Untersuchungen, daß die Gefährdung auf die ersten Lebensjahren beschränkt zu sein scheint, während die Kinder später das Rauchen ihrer Eltern schadlos überstehen.

Schon 1974 kam eine englische Untersuchung an über 2200 Neugeborenen zum Ergebnis, daß ETS das Risiko für Pneumonien und Bronchitis erhöht. Eine Gefährdung wurde damals nur während des ersten Lebensjahres gefunden. Übereinstimmend damit ergab 1985 die „Christchurch, New Zealand Child Development Study“ an 1100 Kindern, daß ETS nur in den ersten zwei Lebensjahren zu vermehrten Symptomen der unteren Atemwege führt und vermehrt Arztbesuche wegen Bronchitis/Pneumonie veranlaßt. In NHANES III wurde ebenfalls eine Tendenz zu vermehrten Peumonien in den ersten beiden Lebensjahren festgestellt.

Bei Asthmaerkrankungen ist die Altersabhängigkeit nicht so deutlich. Zwei Fall-Kontroll-Studien und die National Medical Expenditure Survey von 1987 deuteten zwar darauf hin, daß ETS nur in den ersten Lebensjahren das Auftreten von Asthmasymptomen begünstigt. Nach den Ergebnissen von NHANES III ist das Asthmarisiko aber auch noch im Alter von fünf Jahren erhöht. Dies war jedoch nur nachweisbar, wenn ETS auf die von der Mutter erinnerten Arztdiagnose Asthma bezogen wurde. Die von den Müttern selbst miterlebten Asthmaattacken und „Wheezing“Episoden waren dagegen nur in den ersten beiden Lebensjahren mit ETS assoziiert. Ein Einfluß auf den Schweregrad der Asthmaerkrankung konnte nicht gefunden werden: Die Menge der verordneten Asthmamedikamente war in den Haushalten, in denen geraucht wurde, nicht höher in Familien ohne ETS.

(rme)

Quelle: Gergen PJ et al.: Environmental Tobacco Smoke Exposure on the Respiratory Health of Children 2 Month Through 5 Years of Age in the United States: Third Nationa Health and Nut 101: Exanionation Survey, 1988 to 1994. Pe full/101/2/e8)
Infekte der oberen Atemwege scheinen dagegen nicht durch ETS begünstigt zu werden. 